\section{Microscopic look at prostate cancer}

\section{By Kai-Jye Lou, Staff Writer}

Researchers at Purdue University and Endocyte Inc. have synthesized a series of radioimaging agents that could potentially detect prostate cancer masses and micrometastases that are beyond the detection limit of currently available imaging agents. ${ }^{1,2}$ One compound is expected to enter the clinic by the end of September.

The compounds target prostate-specific membrane antigen (PSMA), which has several properties that make it a good target for molecular imaging agents. The protein is anchored to the cell surface and is not secreted, thus limiting the extent of background interference.

PSMA is also overexpressed in prostate cancer cells compared with that seen in normal prostate tissue. Moreover, its expression in normal prostate tissue is already 100 - to 1,000 -fold higher than that seen in normal nonprostate tissues. ${ }^{3}$

As described in two papers in Molecular Pharmaceutics, research groups led by Philip Low linked a ${ }^{99 \mathrm{~m}} \mathrm{Tc}$-containing imaging fragment to a urea-based, high-affinity PSMAtargeting moiety. The spacer molecule used to link the two fragments was optimized to further increase binding to PSMA. Low is CSO and cofounder of Endocyte and a professor in the
“Low's work was extremely rigorous and I look forward to seeing the clinical translation of these targeted molecular imaging probes."

- Howard Soule, Prostate Cancer Foundation obtain good images in mice after 15 minutes," Low told SciBX. "We anticipate that in humans we will be able to image the patient an hour after treatment."

He added: "The affinity and specificity of our low molecular weight molecules are comparable to an antibody, without the disadvantages associated with antibodies." These disadvantages include poor penetration in solid tumors due to their large size, slow clearance from normal tissues and systemic circulation, and high manufacturing costs.

"Our imaging agents are much smaller than antibodies and they bind to and stay within the tumor," said Low. "If you don't have an agent that binds and stays within the tumor, the compound can leak out into the bloodstream and increase the background radioactivity."

The high tumor affinity of the PSMA-targeting fragment might be further harnessed for therapeutic purposes. As described in one of the Molecular Pharmaceutics papers, the imaging fragment was switched with a derivative of the microtubule inhibitor tubulysin B. In a mouse xenograft model of human prostate cancer, the modified PSMA-targeted compound produced tumor regression with no toxicity-related weight loss.

Thomas Gardner, a urologist and associate professor of urology, microbiology and immunology at Indiana University School of Medicine, highlighted the utility of Low's linker region, which allows for the attachment of either an imaging or therapeutic fragment.

Patrick Peller, an internist and assistant professor of radiology at the Mayo Clinic, thinks the imaging agents described in the Molecular Pharmaceutics articles would have utility in categorizing the disease state of prostate cancer and possibly in assessing response to treatment.

"There's no question that the class of PSMA-targeting compounds is likely to work in prostate cancer imaging," Pomper told SciBX. However, he noted that the compounds developed by his and Low's groups cannot be Department of Chemistry at Purdue.

The ${ }^{99 \mathrm{~m}} \mathrm{Tc}$ radioisotope is the preferred label for use in nuclear medicine. The half-life of ${ }^{99 \mathrm{~m}} \mathrm{Tc}$ is short enough to limit the dose of radiation but long enough to allow sufficient time for any unbound imaging agent to clear from the body, thus increasing image contrast.

The PSMA-targeting fragment is based on a series of high-affinity PSMA inhibitors identified by a group at The Johns Hopkins University School of Medicine led by Martin Pomper. ${ }^{4}$ Pomper, who is a professor of radiology and pharmacology at Johns Hopkins, also has published descriptions of a variety of high-affinity, PSMA-targeted radioimaging agents of the same class, ${ }^{5-8}$ including several that use the ${ }^{99 \mathrm{~m}}$ Tc radioisotope. ${ }^{6}$

What is new in Low's work is the modifications made to the spacer molecule to optimize its fit into a $20 \AA$ pocket of PSMA, as well as the chelator used to bind the radioisotope.

In mice, the low molecular weight compounds accumulated primarily in prostate cancer cells. "With our radioimaging agent, we can compared at this point. "They used a model system with about 10 times more PSMA than" the one used in his own studies, he noted.

\section{Traditional detection}

Imaging is a core component in diagnosing, staging and monitoring prostate cancer. The problem is that the disease is prone to metastasize, and the marketed imaging agents and technologies used to detect these metastases have sensitivity and reliability issues. ${ }^{9}$

"Precise molecular imaging is an unmet medical need for improved prostate cancer diagnosis and for the follow-up of treatment response," said Howard Soule, EVP and CSO at the Prostate Cancer Foundation. "Low's work was extremely rigorous and I look forward to seeing the clinical translation of these targeted molecular imaging probes."

The traditional approach to detect prostate cancer lesions in vivo consists of a bone scan followed by a CT or MRI scan. Bone scans, 
however, have a high false positive rate. CT and MRI scans are generally unable to identify masses smaller than 5-10 $\mathrm{mm}$ and have low sensitivity. ${ }^{9}$

ProstaScint "III-capromab pendetide, a radiolabeled $\mathrm{mAb}$ that recognizes an intracellular region of PSMA, is the only FDA-approved agent to image the extent and spread of prostate cancer.

Although ProstaScint can detect smaller masses than those detected with CT and MRI scans, it has poor sensitivity for bone metastases, which is the first site of metastatic prostate cancer in $72 \%$ of patients. This stems from ProstaScint's inability to bind PSMA on viable cancer cells-the majority of cells in bone metastases remain viable due to the rich blood supply. ${ }^{9}$

EUSA Pharma Inc., which markets ProstaScint, declined to comment.

"There is currently no singly useful imaging compound for prostate cancer," said John Joyal, VP of discovery research at Molecular Insight Pharmaceuticals Inc. He suggested that Low's PSMA-targeted radioimaging agents have "potential to fulfill an unmet clinical need in prostate cancer imaging."

Molecular Insight has evaluated a pair of PSMA-targeted molecular imaging agents in a Phase I trial in patients with metastatic prostate cancer. The company has selected a lead candidate based on the results from the trial and will develop it under the name Trofex.

Peller added that the high affinity of Low's imaging agents would allow for better visualization of smaller masses of cancer cells.

"Better imaging for prostate cancer is going to be huge in guiding therapeutic decisions and preventing patients from receiving unnecessary therapy," said Gardner. "Being able to accurately differentiate when to use surgery and when not to is a very powerful tool."

\section{Avoiding other tissues}

It's less clear whether the compounds will have utility as carriers for therapeutics.

Joyal noted that "Low also shows that the compound is internalized in prostate cancer cells, which is very promising for the design of a radiopharmaceutical" that could treat the disease. He told SciBX the work further validates PSMA as a target for prostate cancer.

On the other hand, Neil Bander, the Bernard and Josephine Chaus Professor of Urologic Oncology and director of Urologic Oncology Research at Weill Cornell Medical College, noted that the low molecular weight approach used by Low will target PSMA wherever it is expressed, including the kidney and the small bowel.

That may be acceptable for imaging, said Bander, but "if you intend to link this small molecule with a cytotoxin, you should expect some renal and/or bowel toxicity."

Low's results showed negligible nephrotoxicity, but Bander wanted to see longer-term safety data. He also wanted to see in-depth biodistribution studies of the imaging agents.

According to Joyal, Low also will need to show how the agents are cleared from the body.

The lead compound from the study has completed GMP manufacturing and is being evaluated as an imaging agent in preclinical toxicity studies. Low said The Purdue Cancer Center and Indiana University Melvin and Bren Simon Cancer Center are funding these studies and preparing the compound for a Phase 0 trial that is expected to begin by the end of September. The trial will evaluate the safety, pharmacokinetics and sensitivity of the imaging agent in prostate cancer patients.

The compounds are available for licensing from the Purdue Office of Technology Commercialization, although Low noted that Endocyte has first rights to license the compounds and is in negotiations with the university. He said the university has filed multiple patent applications covering use of the PSMA-targeted imaging and therapeutic agents in solid tumors, including prostate cancer.

Endocyte's lead compound is EC145, a combination of a folate vitamin analog with a microtubule-destabilizing cancer compound that is in Phase II testing to treat ovarian and non-small cell lung cancer (NSCLC). The trials are also evaluating the company's EC20 molecular imaging agent, which is a combination of a folate vitamin analog and a ${ }^{99 \mathrm{~m}} \mathrm{Tc}$-based imaging agent.

At Johns Hopkins, Pomper told SciBX that imaging PSMA with ureas, including ${ }^{99 \mathrm{~m}} \mathrm{Tc}$-labeled ureas, is covered by multiple issued patents and pending patents. He said the original patent covering imaging agents and methods for imaging PSMA has been licensed to Molecular Insight.

The ${ }^{99 \mathrm{~m} T c-b a s e d ~ P S M A-t a r g e t i n g ~ i m a g i n g ~ a g e n t s ~ d e v e l o p e d ~ b y ~ h i s ~}$ group are available for licensing from Johns Hopkins Technology Transfer.

Lou, K.-J. SciBX 2(17); doi:10.1038/scibx.2009.691

Published online April 30, 2009

\section{REFERENCES}

1. Kularatne, S.A. et al. Mol. Pharm.; published online April 12, 2009; doi:10.1021/mp900069d

Contact: Philip S. Low, Purdue University, West Lafayette, Ind. e-mail: plow@purdue.edu

2. Kularatne, S.A. et al. Mol. Pharm.; published online April 11, 2009; doi: $10.1021 / \mathrm{mp} 9000712$

Contact: Philip S. Low, Purdue University, West Lafayette, Ind. e-mail: plow@purdue.edu

3. Sokoloff, R.L et al. Prostate 43, 150-157 (2000)

4. Pomper, M.G. et al. Mol. Imaging 1, 96-101 (2002)

5. Foss, C.A. et al. Clin. Cancer Res. 11, 4022-4028 (2005)

6. Banerjee, S.R. et al. J. Med. Chem. 51, 4504-4517 (2008)

7. Chen, Y. et al. J. Med. Chem. 51, 7933-7943 (2008)

8. Mease, R.C. et al. Clin. Cancer Res. 14, 3036-3043 (2008)

9. Bander, N.S. Nat. Clin. Pract. Urol. 3, 216-225 (2006)

\section{COMPANIES AND INSTITUTIONS MENTIONED}

Endocyte Inc., West Lafayette, Ind.

EUSA Pharma Inc., Doylestown, Pa.

Indiana University Melvin and Bren Simon Cancer Center, Indianapolis, Ind.

Indiana University School of Medicine, Indianapolis, Ind.

The Johns Hopkins University School of Medicine, Baltimore, Md. Mayo Clinic, Rochester, Minn.

Molecular Insight Pharmaceuticals Inc. (NASDAQ:MIPI),

Cambridge, Mass.

Prostate Cancer Foundation, Santa Monica, Calif.

The Purdue Cancer Center, West Lafayette, Ind.

Purdue University, West Lafayette, Ind.

Weill Cornell Medical College, Ithaca, N.Y. 